\title{
Children Health Promotion during COVID-19 Spread
}

\author{
Fatma A. Abd Elrazek \\ Assistant Professor of pediatric Nursing, faculty of nursing, menoufia University
}

\begin{abstract}
Background: The COVID-19 disrupted the lives children and adults. The virus disrupted life as we know it, the effects of which we are realizing now, Children seem to be the most affected and will endure the long-term impacts of this pandemic. Objective: Identify the impact of pandemic COVID-19 on children health and well-being, identify measures and recommended guidelines to promote children's well -being. Conclusions: The COVID-19 pandemic has a major impact on child's health, mainly physical, social, psychological, and educational aspects. All these implications must be identified and dealt with properly to avoid their consequences, to promote and maintain children's health and well-being.
\end{abstract}

Keywords: COVID-19, COVID-19health consequence, Children's Health promotion.

\section{Introduction:}

Coronavirus disease (COVID-19) pandemic spread and proved rapidly to be a one of the major international health problem. It is a severe acute respiratory syndrome-It spreads very rapidly, through droplets during close and unprotected contact. It can be spread in health care settings by aerosol-generating procedures. Also, it can persist on contaminated surfaces for several days. The most common symptoms include fever, tiredness and a dry cough. Children are less likely to present with these symptoms and may also have, nasal congestion, sore throat, myalgia and diarrhea (Zhu, Zhang\& Wang, 2019)

COVID-19 pandemic produces many sequences on the life and well-being of infants, children, and adolescents. World - wide. Due to the spread of COVID-19, children are more likely to be affected by physical distancing, quarantines, and school closures. Some children feeling more isolated, anxious, bored, and uncertain. They feel fear, and grief, over the impact of the virus on their families (World Health Organization, $2020^{1}$ ).

During COVID- 19 spread pediatric nurse play a critical role in the current situation which, huge amounts of changing information every day, pediatric nurse help the families to keep their children healthy and safe in these uncertain times. Important considerations for children include sharing everyday preventive health behaviors; promoting physical activity; maintaining social connections; watching for signs of stress, anxiety, and depression; and promoting adequate support systems (Society of Pediatric Nurses,2020).

Prevention of COVID-19 
Children with covid-19 disease who have a mild disease course may be isolated at home. While, children with severe disease should be treated in facilities skilled with Particular precautions to protect infants below 1 year of age. WHO recommendations precautions should be followed at health care setting: including risk screening prior to and at entry to clinical sites, separation of children at low and high risk. Health care worker use of protective mouth and nose coverings and protective clothing. Meticulous hand washing and frequent disinfectant use. In areas with a high incidence of infections, families with children should stay at home to reduce social contact for the whole family as much as possible, enhance personal hygiene by regular hand washing, cover coughing with an elbow, and to avoid touching the face (World Health Organization, $2020^{2}$ ).

Infected mothers follow breast feed safely, hand wash, clean the breast and use masks should also be followed in areas with a high incidence of infections; families with children should stay at home to reduce social contact for the whole family as much as possible. Enhance personal hygiene by regular hand washing. Cover coughing with an elbow rather than hands and to avoid touching the face (World Health Organization, 2020 ${ }^{3}$ ).

\section{Vaccines}

Young children be should continue to be seen by a primary care provider for their routine vaccinations and to continue their vaccine as per their usual schedule.
Flu vaccine is more important than ever getting flu vaccination CDC recommends an annual flu vaccine for everyone 6 months and older (US Centers for Disease Control and Prevention, 2020)

\section{Healthcare Delivery and Telemedicine}

Telemedicine service was delivery to meet families' needs during the pandemic with strategies to address disparities in access to telemedicine, and to balance increased access with avoiding harm from missed diagnoses. Telemedicine facilitates use as an alternative to face-to-Face encounter; it may include online visits, live video, mobile health applications and messaging through text or email (World Health Organization, 2020²).

\section{Physical wellbeing}

Their obvious marked change in physical activity as a consequence of COVID-19 spread, which may lead to an increased risk of obesity, diabetes, and cardiovascular disease in children. Programmatic and policy strategies should be geared towards promoting physical activity (Dunton \& Wang, 2020)

It important to stay active. Physical activity promote the immune system, promotes health, and relieves stress, so the recommended guideline for Children ages 3 through 5 years to be active 3 hours /day in different intensity activity, while children and adolescents ages 6 through 17 years should be physically active at least 60 minutes each day. Include aerobic activity (eg. running, jumping , 
muscle-strengthening activities) (American college of sport medicine , 2020: Klein, et al. 2020)

\section{Mental wellbeing}

The COVID-19 pandemic cause an increased in the mental and behavioral health problems among children, because they are experiencing multiple risk factors (e.g., quarantine/isolation, parental stress, social isolation) that could increase the exacerbation of current or the emergence of new mental health problems . Beside, children have experienced increased rates of posttraumatic stress disorder and maybe feeling more isolated, anxious, bored, and uncertain.Also they may feel fear, and grief (Lawson et al.2020). There are some elements related to the pandemic that affects more in children, such as separation from a caregiver, uncertainty about the disease, and the feeling of un support (Li, and Wang, 2020)

The pediatric nurse has an important role in reduction of the impact of COVID-19 on child mental health, they interact with children, their families, thus allowing them to identify mental health problems early and focus on ways to reduce COVID-19 stressors, Nurses can support parents through encourage parent child communication, Reassuring the children, educating them about the situation in age-appropriate ways ,maintaining daily routines, educating children on maintaining safe distances and practicing personal hygiene, acknowledging their distress and answering all their questions with honesty ( Henderson, Schmus, Mcdonald \& Irving, 2020)

\section{Social well being}

The constant fear of contagion affects the daily life of children and leads to social isolation and alter human relations, Social well-being can be promoted during pandemic crisis through keeping children socially connected, reach out to friends and family via supervised phone calls and video chats for their child and their friends, playing games, arts, and listening to music. Promote parent daily routine for share time with children by co-viewing television programs using educational apps. And co-engagement with media (Saladino, Algeri \& Auriemma, 2020).

\section{Education}

After the outbreak of (COVID-19), most countries close the childcare centers, schools, training centers, and higher education facilities, School closures have negative impacts on child health, education, and cognitive development. These closures have affected millions of students worldwide. To ensure continuity in students' learning, and Support intellectual wellbeing, it was recommended to adopt technology in the traditional learning process through offering online (virtual) learning accompanied by other hybrid learning methods (Vlachopoulos, 2020).

\section{Reopening of Schools}

School nurses play an important role during the COVID-19 pandemic in 
reopening schools and child care centers safely for learning, besides supporting other in-person activities. School nurses and other health care providers must routinely evaluate students for symptoms or exposures to COVID -19. They also assist in implementing mitigation strategies; which include contact tracing; maintaining school-based clinics; implementing school-based testing strategies; and supporting students, families, and staff (World Health Organization. $2020^{4}$ ).

The united nations have developed guidance to help countries through the processes, timing, conditions, for reopening schools. The main condition to reopening school is being able to ensure a safe return, by maintaining physical distancing and implementing health measures, such as the use of masks and frequent hand washing (United nation, 2020)

The elementary schools strategies include: Children should wear cloth face coverings, desks should be placed at least 3 feet apart, and ideally 6 feet apart when feasible, cohort classes to minimize crossover among children and utilize outdoor spaces when possible

Secondary schools strategies include : universal face coverings in middle and high schools, particularly when not able to maintain a 6foot distance, avoidance of close physical proximity in cases of increased exhalation (singing)these activities are safest outdoors and spread out, desks should be placed at least 3 feet apart, and ideally 6 feet apart when feasible, cohort classes if possible (UNESCO, UNICEF, 2020)

\section{Conclusions:}

Based on the evidence presented the COVID-19 pandemic disrupted the life of children, with many consequences on children health and well-being .The health care provider and Pediatric Nurses must adopt guideline from World Health organization and Center of Disease control, to enhance health promotion and wellbeing of newborns, infants, young children and adolescence, this guideline carry out children health promotion in several health dimensions, promote physical health, mental ,social and intellectual health.

\section{References:}

American college of sport medicine ( 2020)Keeping Children Active during the Coronavirus Pandemic. Available at https://www.exerciseismedicine.org

Dunton, G. F., Do, B., \& Wang, S. D. (2020). Early effects of the COVID-19 pandemic on physical activity and sedentary behavior in children living in the US. BMC Public Health, 20(1), 1-13.

Henderson, M.D., Schmus, C.J., McDonald, C.C., \& Irving, S.Y. (2020). The COVID-19 pandemic and the impact on child mental health: A socioecological perspective Pediatric Nursing, 46(6), 267-272, 290

Klein JD, Koletzko B,El-ShabrawiMH, et al.Arch Dis Child Epub ahead 
ofprint: [please include

DayMonth Year].

Lawson, M., Piel, M.H., \& Simon, M. (2020). Child maltreatment during the COVID-19 pandemic: Consequences of parental job loss on psychological and physical abuse towards children. Child Abuse \& Neglect, Available at https://doi.org

Li, L. Z., and Wang, S. (2020). Prevalence and predictors of general psychiatric disorders and loneliness during COVID-19 in the United Kingdom. Psych. Res. 291, 0165-

Saladino, V., Algeri, D., \& Auriemma, V. (2020). The psychological and social impact of Covid-19: new perspectives of wellbeing. Frontiers in psychology, 11, 2550.

Society of Pediatric

Nurses,(2020)Information About Corona virus, it available at . http://www.pedsnurses.org/page/ covid-19

US Centers for Disease Control and Prevention (CDC) (2019).

COVID-19 databases and journals. Available: https://www. cdc. gov/ library/

United nation(2020) Policy Brief:

Education during COVID-19 and beyond

UNESCO, UNICEF, (2020),

"Framework for reopening schools", , available at https://unesdoc.unesco.org/ark:/4 $\underline{8223 /}$

Vlachopoulos, D. (2020). COVID-19:

Threat or Opportunity for Online

Education?. Higher Learning

Research Communications, 10 (1).

World Health Organization (WHO). $\left(2020^{1}\right)$ Emergency Committee regarding the outbreak of novel coronavirus (2019-nCoV). 30January 2020. Available at https://www.who.int/news-

World Health Organization. Coronavirus disease $\left(2020^{2}\right)$ Technical guidance :maintaining essential health services and systems,. Available at : https://www. who. int/ emergencies/ disease

World Health Organization $\left(2020^{3}\right)$. Continued breastfeeding for healthy growth and development of children. Available at www.who.int/elena/titles/continu ed

World Health Organization. $\left(2020^{4}\right)$. Corona Virus Disease: Schools/available at www.who.int/emergencies/diseas es/novel

Zhu N, Zhang D, Wang W. (2019) China novel coronavirus investigating and research team. A novel coronavirus from patients with pneumonia in China,. N Engl J Med2020;382:727-3 